\title{
Design-Based Learning: o uso de Objetos Empírico-Concretos e Virtuais na Geometria Descritiva
}

\author{
Design-Based Learning: Using Empirical-Concrete and Virtual Learning Objects in \\ Descriptive Geometry
}

BRUNO, Fernando Batista; Mestre; Universidade Federal do Rio Grande do Sul

fernando.bruno@ufrgs.br

TEIXEIRA, Fábio Gonçalves; Doutor; Universidade Federal do Rio Grande do Sul

fabiogt@ufrgs.br

SILVA, Régio Pierre; Doutor; Universidade Federal do Rio Grande do Sul

regio@ufrgs.br

SILVA, Tânia Luisa Koltermann; Doutora; Universidade Federal do Rio Grande do Sul

tania.koltermann@ufrgs.br

\section{Resumo}

Programas de graduação em Engenharia, Arquitetura e Design oferecem disciplinas como Geometria Descritiva (GD) e Desenho Técnico (DT) em etapas iniciais, para fornecer aos alunos conhecimento básico para lidar com assuntos relativos à representação de projetos ao longo do curso. Entretanto, calouros enfrentam alguns problemas, pois têm pouca ou nenhuma experiência com vistas ortográficas e precisam aplicar esta forma de comunicação ao mesmo tempo que a estão aprendendo. Uma forma de diminuir o nível de abstração destas disciplinas é oferecer recursos empírico-concretos aos alunos, para que possam manipular e analisar objetos tangíveis, em vez de imaginar elementos abstratos. Este artigo propõe uma forma de facilitar a transição entre objetos 3D e vistas 2D, aprimorando o raciocínio e habilidades espaciais dos alunos de GD, a partir do uso de blocos construtivos. Estes blocos são fornecidos no início da disciplina e funcionam como apoio empírico-concreto ao desenvolvimento das atividades propostas em aula.

Palavras Chave: geometria descritiva; objetos de aprendizagem empírico-concretos; aprendizagem baseada em design.

\begin{abstract}
Undergraduate programs in Engineering, Architecture, and Design offer disciplines like Descriptive Geometry (DG) and Technical Drawing (TD) at early stages, to provide students with basic knowledge to deal with subjects related to project representation along the course. However, firstyear students usually face some problems, as they have small (or none) experience with orthographic views and need to learn this form of communication at the same time they are learning it. A way to diminish the level of abstraction of those disciplines is to provide the students with empirical-concrete resources, so they can manipulate and analyze tangible objects, instead of devising abstract elements. This paper proposes a way to ease the transition from 3-D objects to 2$D$ views, leveraging the spatial abilities and reasoning of DG students, using constructive blocks.
\end{abstract}


The blocks are provided at the beginning of the course e work as empirical-concrete support for classroom activities.

Keywords: descriptive geometry; empirical-concrete learning objects; design-based learning. 


\section{Introdução}

A Geometria Descritiva (GD) é uma disciplina extremamente relevante para o desenvolvimento de atividades relacionadas a projeto (TEIXEIRA et al., 2010). A importância da GD em programas de graduação em Engenharia, Arquitetura e Design pode ser verificada de forma direta - o ensino das bases do sistema gráfico de representação de objetos 3D através de vistas 2D, utilizado em projetos, assim como o estudo de sua forma, dimensão e posição - e também indireta - no desenvolvimento e/ou aprimoramento das habilidades espaciais e no raciocínio espacial dos alunos destes cursos (MONTENEGRO, 1991).

Entretanto, o ensino tradicional da GD no Brasil está geralmente apoiado em abordagens abstratas, com base na axiomática (TEIXEIRA et al., 2015), utilizando elementos que dificultam a transposição daquilo que é aprendido em aula para a solução problemas cotidianos dos alunos (TEIXEIRA et al., 2010). Muitos livros norte-americanos (e também brasileiros) sobre representação gráfica em engenharia restringem o assunto "geometria descritiva" a construções elementares, como a determinação da Verdadeira Grandeza de um segmento de reta ou da interseção entre dois polígonos no espaço, por exemplo (STACHEL, 2003). Desta forma, muitas das dificuldades enfrentadas pelos alunos (e principalmente os calouros) "decorrem da carência de apoios empírico-concretos relacionados às operações gráficas realizadas em épura para resolver problemas geométricos" (TEIXEIRA et al., 2015, p. 81).

Outro fator negativo é o posicionamento da GD nas grades curriculares, geralmente nas etapas iniciais dos cursos - nesta fase os alunos têm pouca ou nenhuma experiência com vistas ortográficas. Assim, o volume de reprovação e evasão observados em disciplinas relacionadas a GD costuma ser elevado (SILVA, 2005; SILVA; SILVA; TEIXEIRA, 2007; TEIXEIRA et al., 2006).

O posicionamento da disciplina não pode ser alterado, pois a GD funciona como base para todo o sistema de representação que os alunos utilizarão ao longo de sua carreira. Desta forma, o problema a ser encarado é o tipo de abordagem utilizado no ensino, assim como os tipos de objetos que serão apresentados e trabalhados durante o curso.

O grupo de pesquisa Virtual Design (ViD) tem desenvolvido novas ferramentas e metodologias "com o objetivo de melhorar a qualidade do ensino de GD, buscando a melhoria do processo de aprendizagem dos alunos" (TEIXEIRA et al., 2010). A abordagem adotada pelo ViD é a aprendizagem baseada em design (TEIXEIRA et al., 2006), o que altera a dinâmica das atividades desenvolvidas em sala de aula. Por se tratar de uma metodologia ativa, o foco não está na transmissão do conteúdo, mas na construção coletiva do conhecimento, realizada através da elaboração de soluções para os problemas propostos. Neste ambiente, o professor fornece conceitos básicos e propõe problemas que exigirão o pensamento crítico e a transposição do conhecimento facilitando a compreensão de temas mais específicos.

Quanto ao tipo de objeto a ser trabalhado durante as atividades, o ViD propõe a utilização de elementos sólidos tridimensionais (em vez de ponto, reta e plano), aproximando o ensino da GD à tradição europeia e à sua própria origem, onde a GD não é restrita apenas às projeções ortogonais e métodos descritivos, mas também "abrange técnicas de modelagem de curvas, superfícies e sólidos, oferecendo uma ampla variedade de formas geométricas" (STACHEL, 2003, p. 328-329).

Para apoiar as mudanças propostas, o ViD desenvolveu o HyperCAL $^{3 D}$ (Figura 1), um 
software para modelagem tridimensional que utiliza a linguagem própria da GD para a construção de objetos ao mesmo tempo em que permite sua visualização em 3D e 2D. Além da construção e representação de objetos, o HyperCAL ${ }^{3 \mathrm{D}}$ também oferece ferramentas de manipulação, como a geração de novos planos de projeção e interseção (SANTOS, 2016; TEIXEIRA, 2010; TEIXEIRA; SANTOS, 2014).

Figura 1 - Tela do HyperCAL ${ }^{3 D}$

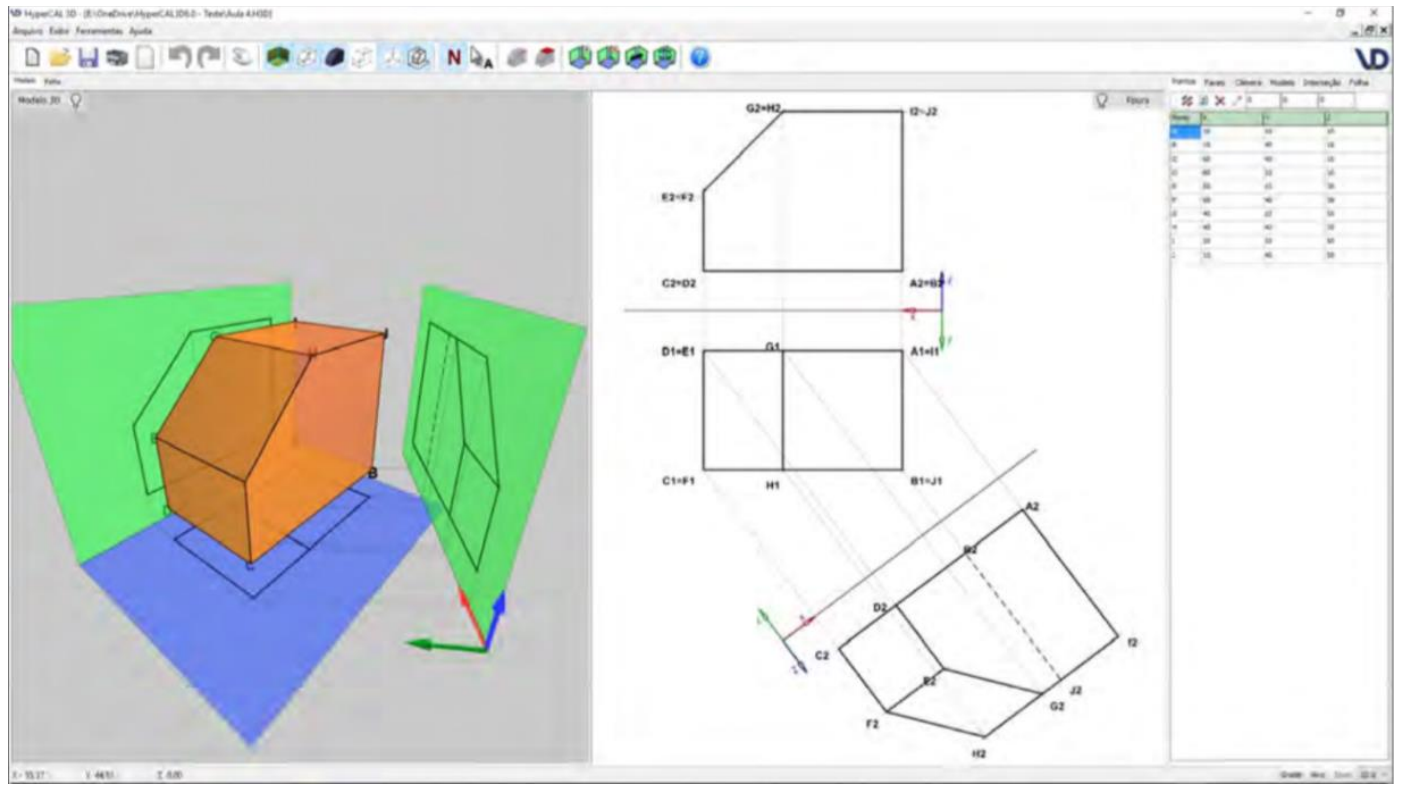

Fonte: Santos (2016, p. 160)

A utilização do HyperCAL ${ }^{3 D}$ "tem proporcionado um aumento significativo da motivação dos alunos para o aprendizado da disciplina, o que tem se refletido na qualidade do aprendizado que fica patente pelo nível dos trabalhos apresentados" (TEIXEIRA et al., 2010, p. 7). O software foi incorporado à disciplina e os alunos desenvolvem seus trabalhos tanto em meio físico (papel e grafite) quanto em meio virtual (HyperCAL ${ }^{3 \mathrm{D}}$ ).

A versão atual do HyperCAL ${ }^{3 D}$, mostrada na Figura 1, apresenta uma área de trabalho híbrida tridimensional e bidimensional, onde o aluno pode realizar as operações tanto no objeto 3D, quanto na sua representação 2D (épura). As operações são realizadas de forma transparente e a correspondência das ações é imediata entre os ambientes $2 \mathrm{D}$ e $3 \mathrm{D}$ - o que é feito na épura ocorre ao mesmo tempo no 3D e vice-versa.

\section{Desenvolvimento dos objetos}

O desenvolvimento dos objetos de aprendizagem empírico-concretos foi baseado nos exercícios de percepção espacial propostos no livro "Inteligência Visual e 3-D" (MONTENEGRO, 2005). Os objetos permitem a construção de elementos com diferentes configurações geométricas, geradas a partir de diversas combinações possíveis, que podem obedecer, a critério do professor, a um conjunto de requisitos e restrições. Este recurso é formado por um conjunto de cinco blocos modulares, obtidos através de transformações realizadas em um cubo (Figura 2). 

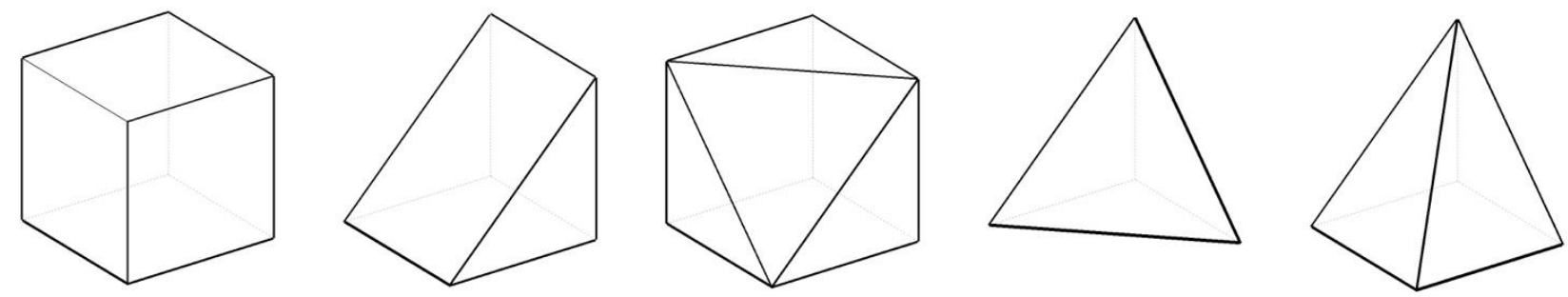

Fonte: os autores

Para que as peças pudessem ser construídas de forma simples, o material utilizado é o papel Smith (embora um conjunto tenha sido confeccionado em madeira, o que demandou mãode-obra especializada) e cada módulo mede 50 × 50 × 50 (mm).

O conjunto de blocos permite que os alunos experimentem, de forma prática, as diferentes posições dos objetos em relação ao Sistema de Projeção - é possível a obtenção das 7 posições particulares de faces (frontal, horizontal, de perfil, vertical, de topo, rampa e oblíqua) e das 7 posições particulares de arestas (vertical, de topo, fronto-horizontal, perfil, horizontal, frontal e oblíqua) em relação ao diedro.

A forma de cada um dos blocos foi projetada segundo os critérios de simplicidade (no total, o conjunto apresenta apenas 5 geometrias diferentes de face e 3 de aresta) e modularidade (as peças "aderem" umas às outras graças à baixa variedade de geometrias de faces e arestas). Os protótipos utilizados até o momento ainda não apresentam sistema de encaixe, o que já está sendo pesquisado para versões futuras dos objetos.

Os blocos permitem, também, que os alunos analisem a relação entre a posição de um elemento e o tipo de projeção gerado (verdadeira grandeza, projeção reduzida e projeção acumulada), além de permitir o estudo de posições relativas entre os elementos e torna mais familiar o uso dos sistemas de coordenadas para referenciar a representação gráfica de modelos tridimensionais.

Porém, as contribuições mais relevantes deste material de apoio estão na compreensão, através da reflexão na ação e na manipulação de formas concretas, das relações abstratas entre a geometria dos objetos no espaço tridimensional e a sua representação no sistema de dupla projeção.

\section{Utilização dos objetos}

O objetivo das atividades envolvendo os blocos é auxiliar o aluno a percorrer algumas etapas do projeto geométrico de formas tridimensionais, formando um ciclo de atividades que inclui a modelagem, a representação, alteração, planificação, prototipagem e avaliação, conforme apresentado na Figura 3 (o ciclo de atividades está representado de forma simplificada para manter a legibilidade da figura, pois durante o processo de projeto há diferentes níveis de interação entre as etapas, com ciclos recorrentes ou mesmo subciclos que ocorrem em determinados conjuntos de etapas). Durante a execução das etapas propostas, há passagens do 3D para 2D e do 2D para o 3D, o que estimula a compreensão dos processos de representação e dos métodos descritivos da GD. 
Figura 3: ciclo de atividades envolvendo os blocos

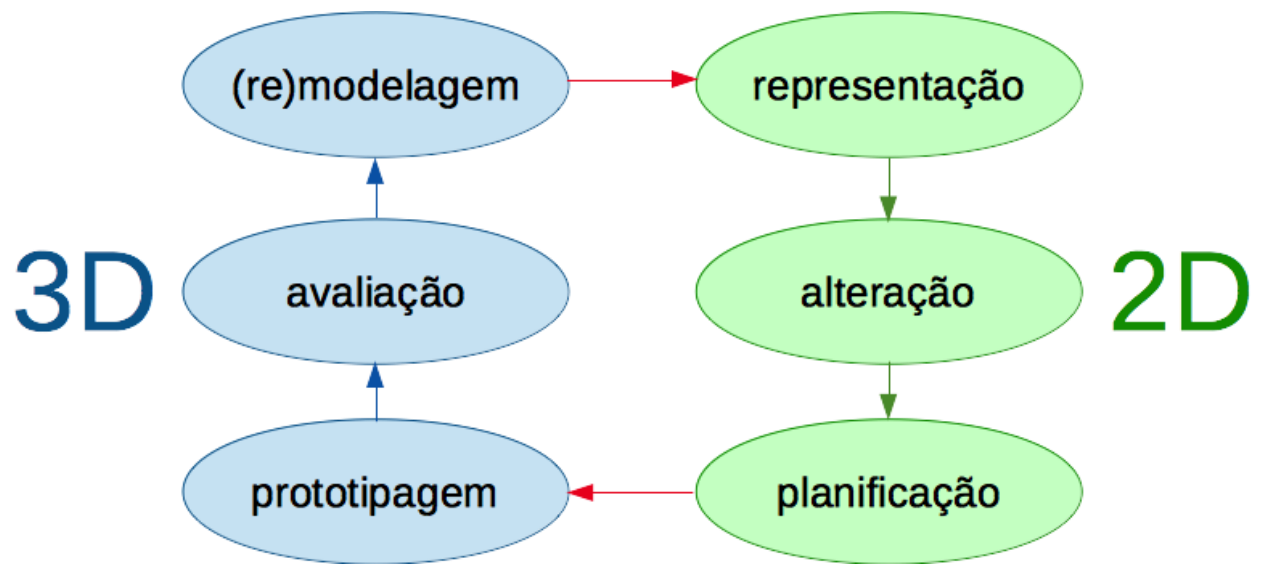

Fonte: os autores

O material tem sido utilizado de duas maneiras:

1) 2D $\Rightarrow$ 3D (exercício de leitura): os alunos recebem projetos representados em épura (Figura 4A) e são solicitados a montar o objeto tridimensional a partir da combinação de blocos (Figura 4B). Este tipo de exercício é realizado de forma rápida e tem sido aplicado durante a etapa introdutória da disciplina, onde são tratados conceitos como sistemas de projeção, projeção ortogonal cilíndrica e sistemas de coordenadas. Este tipo de exercício tem se mostrado eficiente para a compreensão de conceitos fundamentais do sistema de representação da GD.

Figura 4 - Exercício de leitura de vistas ortográficas

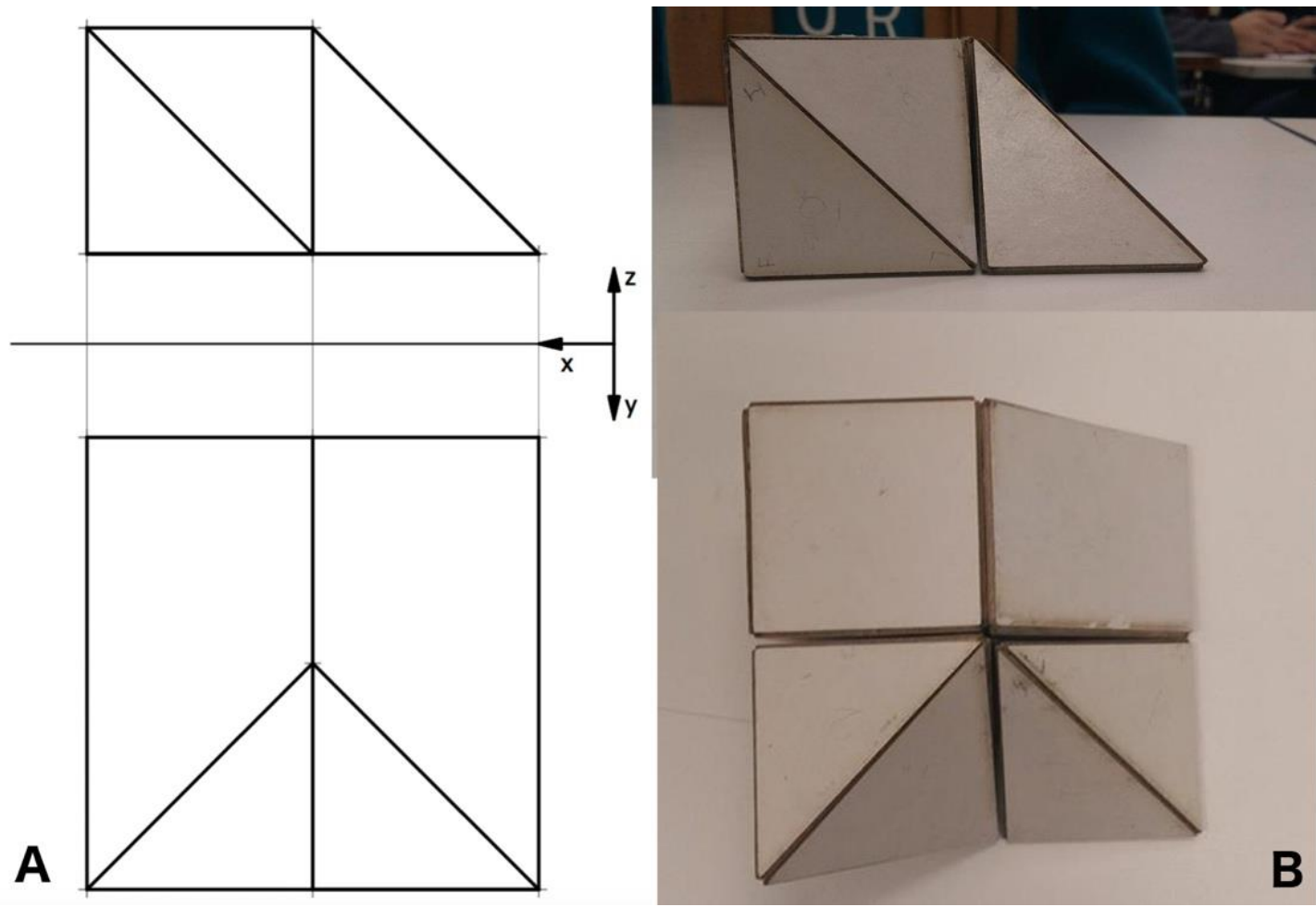

Fonte: os autores 
2) $3 D \Rightarrow 2 D$ (exercício de modelagem): os alunos recebem um conjunto de blocos para projetarem e construírem um objeto partir de combinações entre os blocos, apoiados em uma lista de requisitos e/ou restrições. Neste tipo de exercício, os alunos começam a trabalhar a representação do objeto a partir de uma referência física, que eles próprios projetaram e construíram. Esta é uma atividade mais demorada, e tem sido realizada ao longo da disciplina, seguindo uma sequência de fases, que evolui conforme os assuntos são abordados em aula:

a) Modelagem: Projetar e construir um objeto sólido com determinadas características (apresentar determinados tipos de projeção de faces ou de arestas, por exemplo), utilizando, ao menos, um bloco de cada tipo ou uma quantidade mínima (e/ou máxima) de blocos (Figura 5);

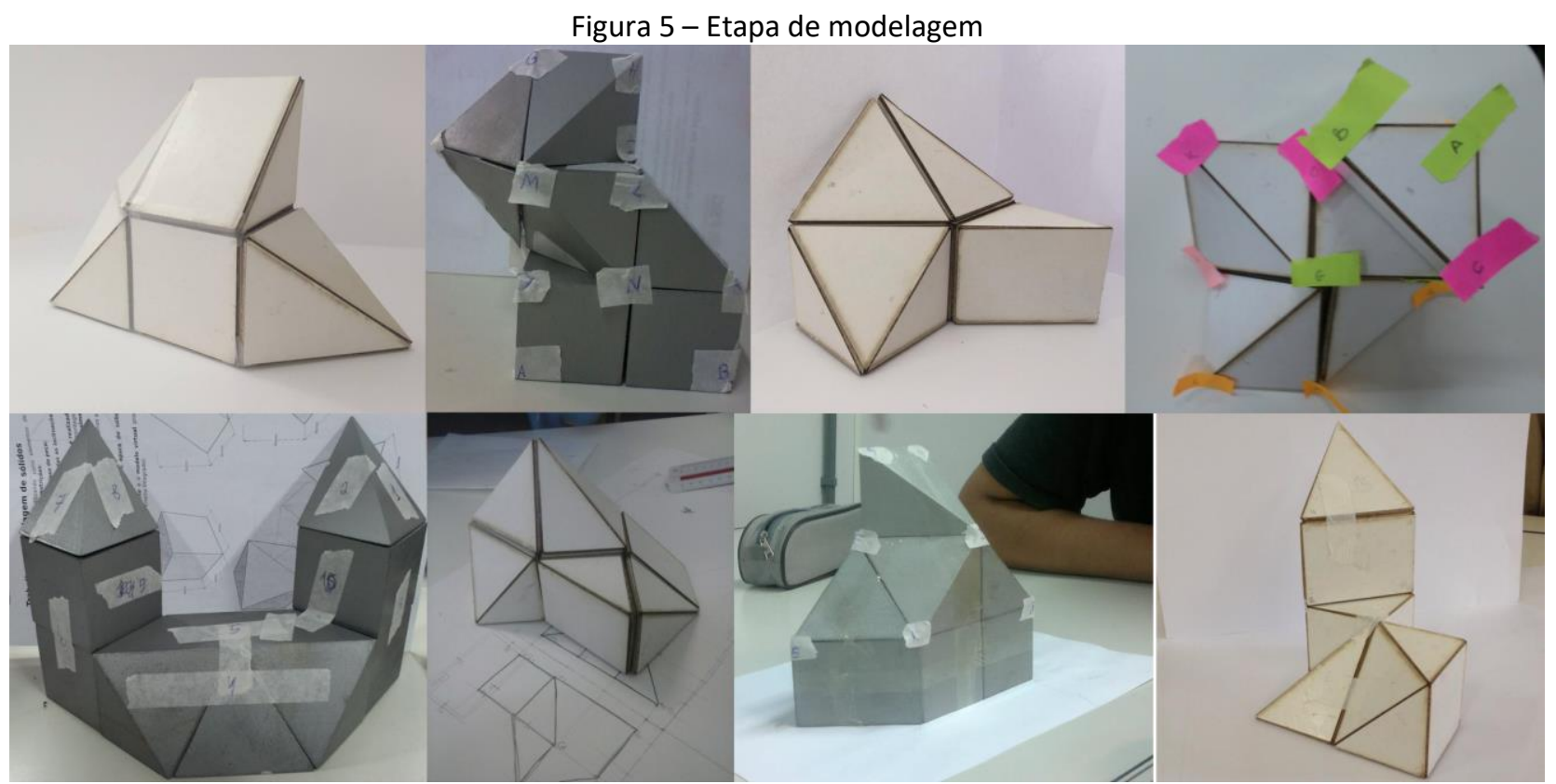

Fonte: os autores

b) Representação: Antes da etapa de representação, os alunos devem obter as informações necessárias para o projeto, o que envolve identificar os vértices do objeto, posicioná-lo no diedro, obter suas coordenadas e estabelecer as conectividades das faces do objeto (Figura 6). A partir destas informações, o objeto é representado em épura e em meio virtual através do HyperCAL3D;

Figura 6 - Exercício de projeto, representação e construção

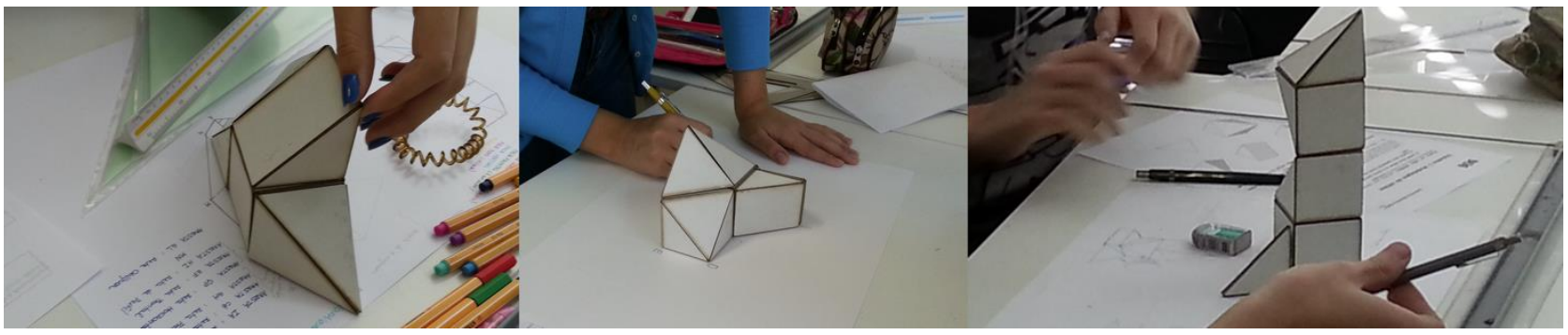

13 Congresso Brasileiro de Pesquisa e Desenvolvimento em Design, Univille, Joinville (SC) 
c) Edição: A partir das projeções do objeto, cortá-lo ou realizar a extrusão de uma ou mais faces, alterando sua forma;

d) Planificação e prototipagem: Planificar as faces do objeto, gerando um projeto de dobra e corte para a construção do modelo físico (Figura 7).

Figura 7 - Planificação e construção

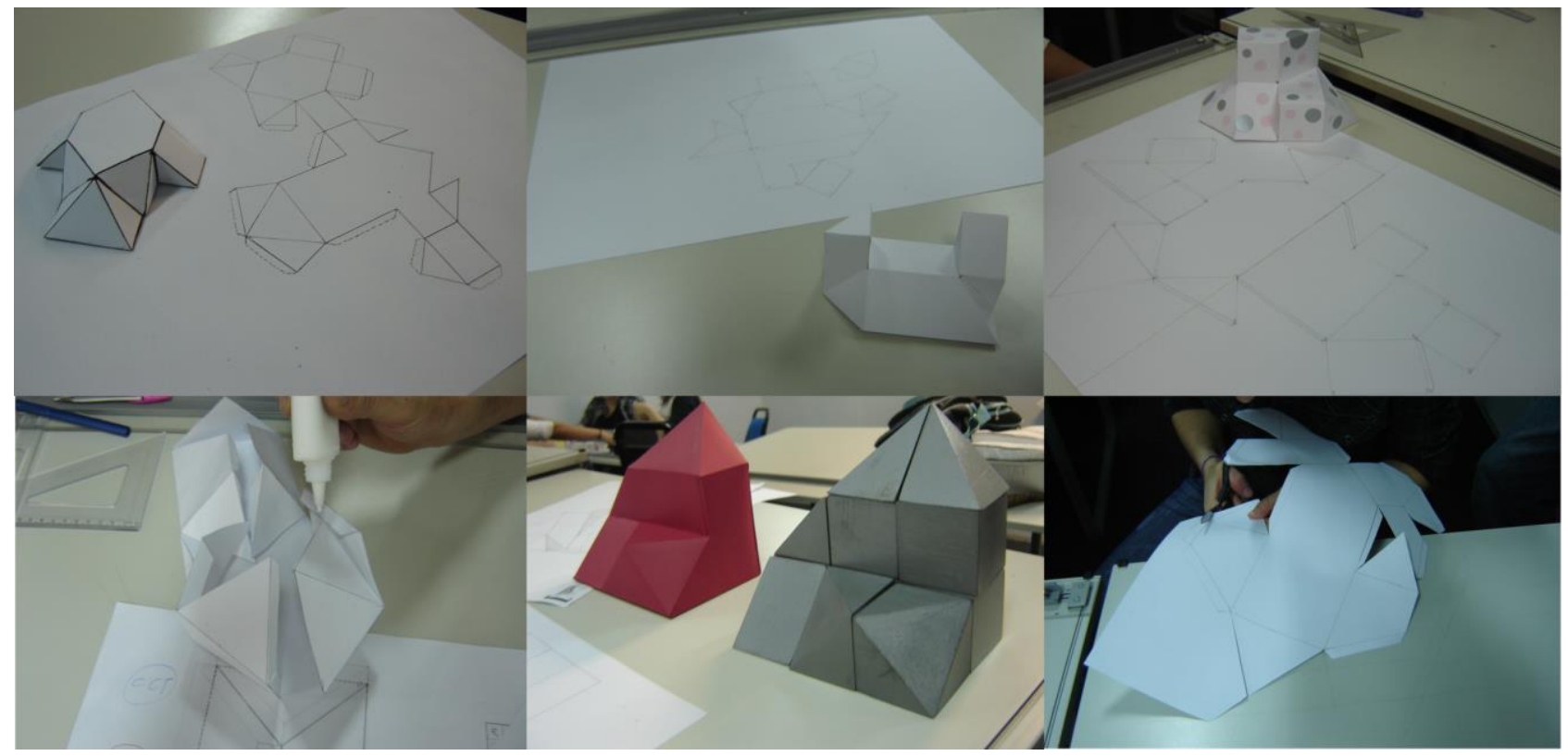

Fonte: os autores

\section{Discussão}

As atividades propostas com os modelos físicos se mostraram extremamente produtivas do ponto de vista didático, promovendo uma integração dos conteúdos estudados, além de uma integração e colaboração entre os estudantes, criando um ambiente de solidariedade e propício ao processo de aprendizagem.

A etapa de modelagem é uma etapa criativa onde os alunos realizam em grupo o projeto geométrico de um sólido cujos requisitos são estabelecidos pelo professor, como por exemplo, conter determinados posições de arestas e faces. Nesta etapa, os alunos relacionam os conteúdos referente às posições de retas e planos com o sistema de dupla projeção e podem assim compreender o seu significado prático e as formas concretas associadas às referidas posições de arestas (retas) e faces (planos).

$\mathrm{Na}$ etapa de representação, os estudantes devem interpretar o modelo físico concreto para gerar a sua representação abstrata em um sistema de coordenadas e um conjunto de conectividades. Este processo exercita o raciocínio lógico espacial ao mesmo tempo que facilita a passagem do concreto para o abstrato, pois as relações entre os mesmos passam a ser facilmente perceptíveis. Por outro lado, o uso do software HyperCAL ${ }^{3 D}$ facilita a compreensão destas relações, pois permite a visualização em tempo real dos modelos representados e de suas 
projeções.

A etapa de edição permite a utilização dos conceitos de mais complexos de GD, como interseção e extrusão, para permitir o projeto de um objeto mais complexo a partir do sólido gerado na etapa de modelagem. Assim, a partir da representação em projeções, são realizadas as operações de GD necessárias para cortar o sólido e, eventualmente, realizar a extrusão de uma determinada face, gerando, assim, uma geometria mais complexa e elaborada com base na geometria do modelo original. Este processo é realizado sobre as projeções e pode realimentar a representação tridimensional, atualizando o modelo virtual no HyperCAL ${ }^{3 \mathrm{D}}$, o que promove uma melhor compreensão das alterações formais resultantes dos processos de corte e extrusão, pois o modelo pode ser visualizado de qualquer posição a partir de uma câmera virtual.

A etapa planificação aplica os conceitos de GD para planificar a superfície do sólido para permitir a construção do modelo físico da nova geometria gerada. São utilizados os conceitos de Mudança de Sistema de Referência (MSR) (ou Mudança de Plano), Rotação e Rebatimento para encontrar a Verdadeiras Grandezas (VGs) das faces e, assim, para montar um projeto de recorte e dobra em papel para a construção do modelo físico. Estas operações de MSR podem ser feitas diretamente no HyperCAL ${ }^{3 \mathrm{D}}$ (Figura 8) ou no papel. A prototipagem é realizada através do recorte, dobras e colagem das bordas conforme o projeto.

Figura 8 - Planificação da superfícies de um sólido utilizando MSR no HyperCAL ${ }^{3 D}$

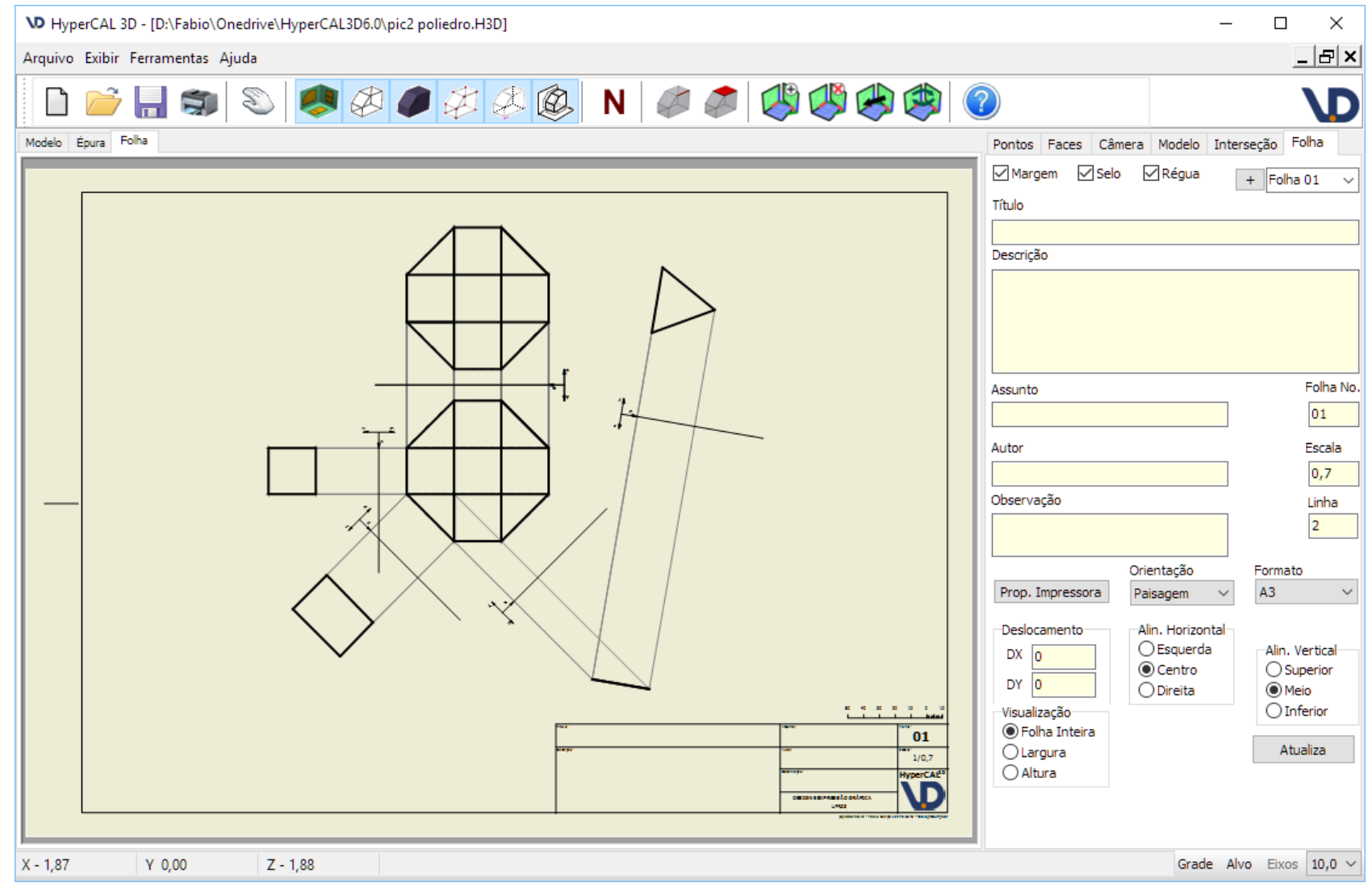

Fonte: os autores

$\mathrm{Na}$ (re) modelagem, o modelo físico pode ser novamente modificado por cortes e 
realimentar todo o processo, retornando para a representação e, assim, sucessivamente. Neste ciclo de atividades, fica evidente a passagem do concreto para o abstrato, do 3D físico para o 3D virtual, do 3D virtual para o 2D virtual, do 2D físico para o 3D físico e, durante todo o ciclo a realização das operações da GD para resolver os problemas de projeto.

\section{Considerações Finais}

O uso de ferramentais computacionais para auxiliar no processo de ensino-aprendizagem é uma tendência contemporânea e que faz todo o sentido diante dos avanços tecnológicos das últimas duas décadas nesta área. No entanto, o uso de modelos físicos, que é uma prática que tem sido sistematicamente abandonada, pode acrescentar qualidades que o uso exclusivo dos ambientes virtuais não proporciona. Assim este trabalho apresentou uma forma de unir o melhor destas duas possibilidades para melhorar o processo de aprendizagem da Geometria Descritiva sob a ótica do projeto geométrico de sólidos facetados.

Uma abordagem que utiliza um ciclo de etapas, onde o processo criativo de projeto utiliza o apoio empírico-concreto de modelos físicos, passando por etapas de representação virtual e analógica, até retornar aos modelos físicos, mostrou-se extremamente promissora para mediar a transposição do concreto para o abstrato, uma competência fundamental para um projetista, mas que é uma das maiores dificuldades no processo de aprendizagem da Geometria Descritiva. O uso de recursos multimodais integrados sob uma metodologia consistente integra os conhecimentos de GD aos conhecimentos prévios dos alunos, além de promover um grande engajamento nas atividades, o que é uma característica deste tipo de abordagem hands on.

\section{Referências}

MONTENEGRO, G. A. Geometria descritiva. São Paulo: Edgard Blucher, 1991.

MONTENEGRO, G. A. Inteligência Visual e 3-D: Compreendendo Conceitos Básicos da Geometria Espacial. São Paulo: Blucher, 2005.

SANTOS, S. L. Interface interativa bidimensional em um software para o ensino de geometria descritiva. 2016. Doutorado - Universidade Federal do Rio Grande do Sul, Porto Alegre, 2016. Disponível em: <http://hdl.handle.net/10183/152710>. Acesso em: 20 fev. 2018.

SILVA, R. P. Avaliação da Perspectiva Cognitivista Como Ferramenta de Ensino-Aprendizagem da Geometria Descritiva a Partir do Ambiente Hipermídia HyperCAL GD. 2005. 213 f. Tese de Doutorado - Universidade Federal de Santa Catarina, Florianópolis, 2005. Disponível em: <https://repositorio.ufsc.br/handle/123456789/101609>. Acesso em: 7 jul. 2009.

SILVA, R. P.; SILVA, T. L. K.; TEIXEIRA, F. G. Learning objects: an approach in engineering education in a cognitive perspective. In: INTERNATIONAL CONFERENCE ON ENGINEERING AND EDUCATION 2007, 2007, Coimbra. Anais... Coimbra: [s.n.], 2007. Disponível em: <http://hdl.handle.net/10183/31081>. Acesso em: 15 fev. 2017.

STACHEL, H. What is descriptive geometry for? . Viena: Institute of Discrete Mathematics and Geometry, TU Wien, 2003. Disponível em: <http://www.geometrie.tuwien.ac.at/stachel/dresden.pdf>. Acesso em: 8 jan. 2016. 
TEIXEIRA, F. G. et al. Experiências inovadoras em ensino e pesquisa da Geometria Descritiva. Revista Brasileira de Expressão Gráfica, v. 3, n. 2, p. 80-92, 2015. Disponível em: <http://rbeg.net/artigos/artigo29.pdf>. Acesso em: 21 fev. 2018.

TEIXEIRA, F. G. et al. Geometria Descritiva: uma abordagem concreta. In: XXXVIII CONGRESSO BRASILEIRO DE EDUCAÇÃO EM ENGENHARIA, 2010, Fortaleza. Anais... Fortaleza: [s.n.], 2010.

TEIXEIRA, F. G. et al. The descriptive geometry education through the design-based learning. 2006, [S.I: $\quad$ S.n.], 2006. Disponível em: <http://www.researchgate.net/profile/Fabio_Teixeira4/publication/233910688_THE_DESCRIPTIVE _GEOMETRY_EDUCATION_THROUGH_THE_DESIGN_BASED_LEARNING/links/Ofcfd50cc6a53381b0 000000.pdf>. Acesso em: 8 jan. 2016. 